\title{
Relationship of the Openness Personality Trait and Development of Belief in God
}

\section{Anne W. Njenga}

\section{Background}

The development of an individual's beliefs about God is significantly influenced by teachings of parents.

However, not all children have the exact same beliefs as their parents.

This study examined what may be the characteristics of individuals who have similar or differing beliefs than those to which they were exposed as a child.

Prior research has found that:

- Openness to experience was the personality characteristic that differed most between the religious and non-religious respondents. (Caldwell-Harris, 2012)

- Even non-religious people can hold very strong views and stick to these opinions avidly. (McCrea et al, 1999)

- Beliefs in emerging adulthood show an increase in a skeptical view of religious institutions. (Arnett, Jensen (2002)

The adult's beliefs about God are proposed to be a combination of the set of ideas taught by the parent and the personality characteristic of the adult child, which have allowed the individual to retain or migrate away from those teaching.

References

Arnett, J. J. (2002). Emerging adulthood: A theory of development from the late teens through the twenties. American Psychologist, 55, 469-480

Caldwell-Harris, C. L. (2012). Understanding atheism/non-belief as an expected individual-difference variable. Religion, Brain and Behavior, 2(1), 4-23

McCrae et al (1999). Age differences in personality across the adult life span: Parallels in five cultures. Developmental Psychology, 35, 466-477

\section{Hypothesis}

Adults who score higher in Openness (on the Big Five Personality Traits) are more likely to have a set of beliefs about God that are dissimilar to the ideas which were taught to them as a children.

\section{Methods}

PARTICIPANTS

- 26 females \& 14 males; 30 to 65 years old

- All - family and friends of researcher

- Primarily - Residents of Kenya, Africa (see Note below)

MATERIALS

Big Five Personality Inventory - provided evaluation of an individuals levels of Openness, Conscientiousness Extroversion, Agreeableness and Neuroticism.

Similarity of Beliefs Questionnaire - 11-item checklist that evaluated an individual's current/adult beliefs about God; the same 11 questions were used to detail the family beliefs that the person was taught as a child.

The "Similarity of Beliefs Score" was determined equal to the total number of items that were identical for the participant's current beliefs and those taught by family when growing up.

PROCEDURE

- Participants were contacted by email and Facebook.

- Questionnaire materials were completed anonymously at an online survey site.

Notes - Re. KENYAN SOCIETY: Primarily - Christian faith; over $80 \%$ are very religious people.

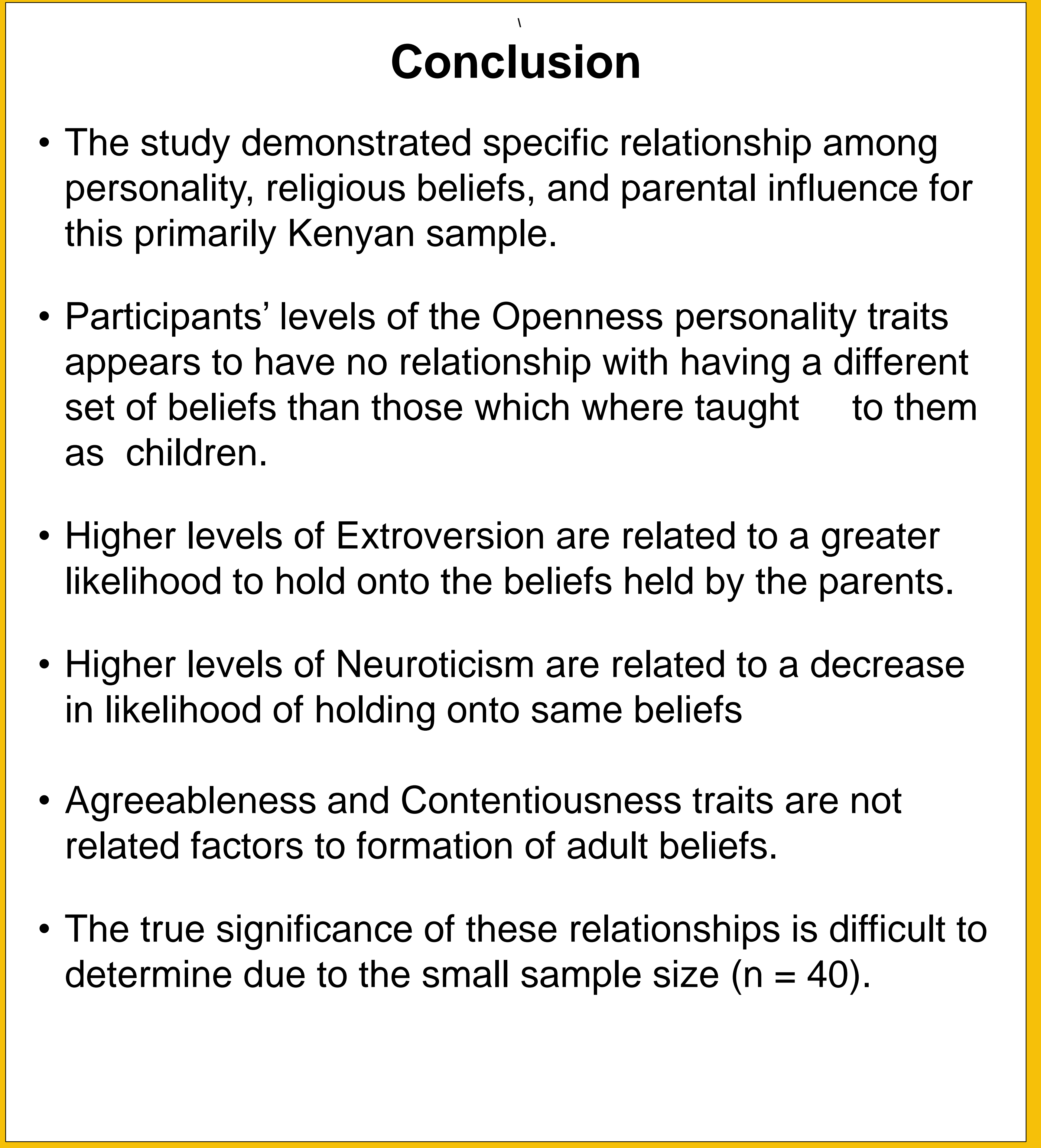

Results

(Pearson Correlational Analysis)

TEST OF HYPOTHESIS

Higher Openness was found with greater Similarity of Beliefs Not significantly related: $\quad r=+0.24 \quad \mathrm{p}=14 \%$ Hypothesis is not supported.

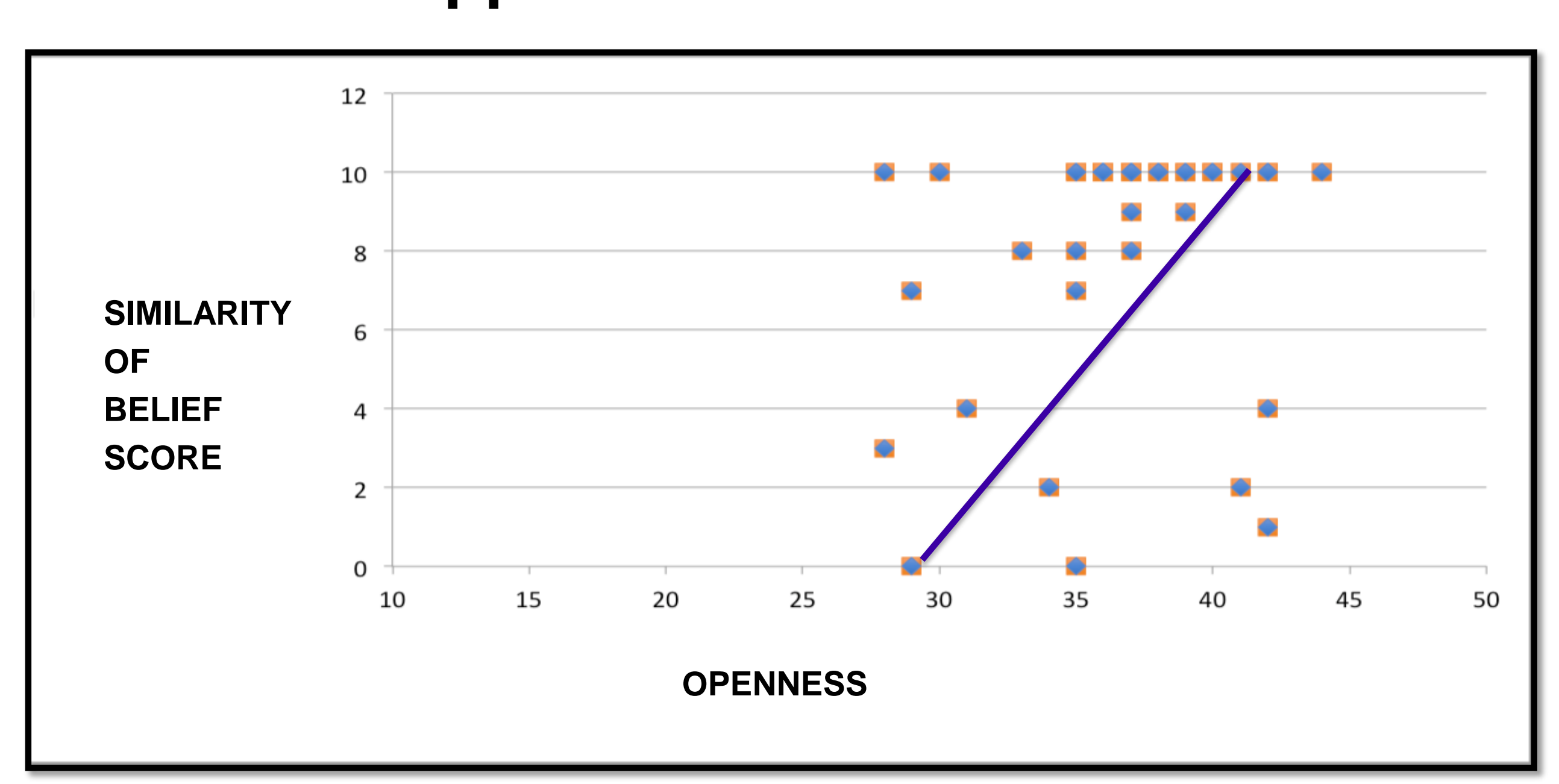

EVALUATION OF OTHER PERSONALITY TRAITS

Traits with significant relationship to Similarity of Beliefs

Extroversion: $\quad r=+0.28 \quad p=4.7 \%$

Neuroticism: $\quad r=-0.29 \quad p=3.9 \%$

Traits with NO significant relationship to Similarity of Beliefs

Agreeableness: $\quad r=+0.15 \quad p=32 \%$

Conscientiousness: $\quad r=+0.19 \quad p=18 \%$ 